\title{
Study of Heat Flux Sensor Test Containing Metal Phase Change Heat Sink
}

\author{
Xiao-Heng DING ${ }^{1, a}$, Song-He MENG ${ }^{2, b,{ }^{*}}$, Fa-Jun $\mathrm{YI}^{3, \mathrm{c}}$, Yan-Wei ZHU ${ }^{4, \mathrm{~d}}$ \\ ${ }^{1,2,3,4}$ Center for Composite Materials and Structure, Harbin Institute of technology, No.2 Yikuang Str, \\ harbin 15008, china \\ adingxiaoheng267@126.com, ${ }^{b}$ mengsh@hit.edu.cn, ${ }^{\text {cf }}$ ajun_yi@126.com, ${ }^{d}$ zhuyanwei5@163.com \\ ${ }^{*}$ Corresponding author
}

Keywords: Phase change materials, Heat flux sensor, Integrated preparation, Heat flux calibration, Heat sink efficiency characterization.

\begin{abstract}
Heat flux is a key parameter to characterize energy transport process in the fields of energy, metallurgy and aerospace. Traditional heat flux sensor can bear load and guarantee normal work of instrument through heat capacity or air cooling and water cooling system of own materials. However, application environment in the aerospace field has many limitations on instrument structure shape and cooling mode. The efficiency of Phase Change Materials (PCM) is higher than traditional quality heat sink by one order of magnitude, which is much simpler compared with air-cooled, water-cooled and other additional system structure. In the paper, $\mathrm{Sn}-\mathrm{Bi}$ alloy with low melting point was regarded as phase change material, copper foam metal was used as heat transfer reinforcement skeleton, an integrated structure of sensor/PCM heat sink was prepared through vacuum infiltration. Halogen lamp equipment was used as radiation heat source. Responses of two sensors of oxygen free copper traditional structure and composite structure containing PCM were compared under the same heat flux load. Experiment proved that weight of composite structure containing PCM was reduced by 16.8g under nearly identical thermal load. Constructed function $\mathrm{Kj}$ was adopted for characterizing heat sink efficiency of sensor. Heat sink efficiency mutation of sensor containing PCM exceeded control sensor in the interval of alloy phase change temperature range.
\end{abstract}

\section{Introduction}

Heat flux refers to energy passing through unit area within unit time in the heat transfer process, which is regarded as parameter for characterizing energy transport order in various heat exchange processes and key benchmark of various thermal designs in aerospace, energy power, metallurgy and other industries. Heat flux sensor is usually based on certain heat transfer model, heat flux acted on sensitive element thereof is converted into linear or nonlinear electrical signal for output. It can bear own temperature change after energy input and output aiming at heat flux sensor with short time and low range, for example it can be applied in soil heat flux measurement or building wall thermal insulation assessment[1 2]. Although flow field environments of some aeronautics and astronautics wind tunnel equipment can reach $\mathrm{MW} / \mathrm{m}^{2}$ magnitude[3], but test model is usually only exposed for a few seconds or a few milliseconds, and the adopted heat flux sensor also can bear load through heat capacity of own structure. However, sensor capable of being applied under higher order condition for long term should be kept in certain allowable temperature range, thereby ensuring geometric stability and physical stability of sensor structure and materials. More importantly, one-dimensional or two-dimensional model assumption is adopted for most heat flux sensors[4]. Therefore, effective energy channel is important premise to ensure physical model and boundary conditions in measuring principle under the condition of load, which also acts as precondition of measurement accuracy. For example, cooling water should be turned on during long-term application of circular foil heat flux sensor (Gardon-Gage) [5] and other equipment, and allowable temperature of the equipment can be kept by liquid circulation. Commonly surface measurement and assessment aerodynamic heat of aircraft is important target parameters of flight test[6]. The sensor has strict requirements on 
equipment dimension and weights. Additional cooling equipment may seriously reduce load efficiency of aircraft, therefore 'quality heat sink' is mostly adopted for measurement devices of aircraft. Heat flux measurement device is designed in a targeted mode according to flight load, such as equipment in literature [7][8], the shape and quality must ensure that the temperature rise of sensor structure is within the safety scope under the application environment.

Phase change materials can absorb a lot of heat with smaller temperature rise $\Delta \mathrm{T}$ in phase change temperature range by the aid of own phase change latent heat. Phase change heat sink has high efficiency advantages compared with quality heat sink. Various metal phase change materials mainly composed of alloys with low melting point has larger density compared with inorganic phase change materials [9]. It has prominent advantages and application potential under the requirements of aerospace on integration, miniaturization and high reliability. In the study, bismuth-tin alloy was adopted as phase change materials, foam copper is adopted as thermal conductivity enhancement skeleton, $\Phi 16 \times 20 \mathrm{~mm}$ phase change heat sink slug was prepared through vacuum infiltration in heat flux sensor structure. Halogen lamp heater was adopted as radiation heat source. Constant heat flux was adopted for respectively loading sensor containing phase change heat sink and sensor of traditional quality heat sink. Measurement results of the two sensors were compared for evaluating structure efficiency.

\section{Research Methods and Experiment Design}

\section{Theoretical Basis of Heat Flux Measurement}

A simple method was proposed in literature [10] aiming at transient one-dimensional inverse heat conduction problem, namely: it was believed that temperature $T(x, t)$ of in transient one-dimensional problem at any time $t$ and any location $x$ can be expressed by cubic polynomial about coordinate $x$ as shown in formula (1), wherein polynomial coefficient was a function of time $t$.

$$
T(x, t)=\sum_{n=0}^{3} C_{n}(t) x^{n}
$$

If positions of two points $x_{1}$ and $x_{2}$ as well as temperature time histories $T\left(x_{1}, t\right)$ and $T\left(x_{2}, t\right)$ were known in control volume of one-dimensional problem. Temperature and the second order partial derivative of temperature on position were expressed with formula (1), and the equation is expressed as follows in matrix form:

$$
\left[\begin{array}{c}
T\left(x_{1}, t\right) \\
T\left(x_{2}, t\right) \\
\frac{\partial^{2} T\left(x_{1}, t\right)}{\partial x^{2}} \\
\frac{\partial^{2} T\left(x_{1}, t\right)}{\partial x^{2}}
\end{array}\right]=\left[\begin{array}{cccc}
1 & x_{1} & x_{1}^{2} & x_{1}^{3} \\
1 & x_{2} & x_{2}^{2} & x_{2}^{3} \\
0 & 0 & 2 & 6 x_{1} \\
0 & 0 & 2 & 6 x_{2}
\end{array}\right]\left[\begin{array}{l}
C_{1}(\mathrm{t}) \\
C_{2}(\mathrm{t}) \\
C_{3}(\mathrm{t}) \\
C_{4}(\mathrm{t})
\end{array}\right]
$$

It was recorded as follows:

$$
A=B C
$$

The second order derivative of temperature on position in column vector on the left of the equation (2) was added into Fourier law (3),

$$
\frac{\partial^{2} T}{\partial x^{2}}=\frac{1}{\alpha} \frac{\partial T}{\partial t}
$$

Both sides of the equation are continuously multiplied by inverse matrix of $\mathrm{B}$, vector of polynomial coefficients $C n(t)$ in equation (1) can be expressed as follows: 


$$
C=B^{-1} A
$$

Wherein:

$$
\begin{aligned}
& B^{-1}=\left[\begin{array}{cccc}
\frac{x_{2}}{x_{2}-x_{1}} & \frac{-x_{1}}{x_{2}-x_{1}} & \frac{x_{1} x_{2}\left(2 x_{2}-x_{1}\right)}{6\left(x_{2}-x_{1}\right)} & \frac{x_{1} x_{2}\left(x_{2}-2 x_{1}\right)}{6\left(x_{2}-x_{1}\right)} \\
\frac{-1}{x_{2}-x_{1}} & \frac{1}{x_{2}-x_{1}} & \frac{x_{1}^{2}-2 x_{1} x_{2}-2 x_{2}^{2}}{6\left(x_{2}-x_{1}\right)} & \frac{2 x_{1}^{2}+2 x_{1} x_{2}-x_{2}^{2}}{6\left(x_{2}-x_{1}\right)} \\
0 & 0 & \frac{x_{2}}{2\left(x_{2}-x_{1}\right)} & \frac{-x_{1}}{2\left(x_{2}-x_{1}\right)} \\
0 & 0 & \frac{-1}{6\left(x_{2}-x_{1}\right)} & \frac{1}{6\left(x_{2}-x_{1}\right)}
\end{array}\right] \\
& A=\left[\begin{array}{c}
T\left(x_{1}, t\right) \\
T\left(x_{2}, t\right) \\
\frac{\partial^{2} T\left(x_{1}, t\right)}{\partial x^{2}} \\
\frac{\partial^{2} T\left(x_{1}, t\right)}{\partial x^{2}}
\end{array}\right]=\left[\begin{array}{c}
T\left(x_{1}, t\right) \\
T\left(x_{2}, t\right) \\
\frac{1}{\alpha} \frac{\partial T\left(x_{1}, t\right)}{\partial t} \\
\frac{1}{\alpha} \frac{\partial^{2} T\left(x_{1}, t\right)}{\partial t}
\end{array}\right]
\end{aligned}
$$

All elements in the matrix were functions of coordinates $x_{1}$ and $x_{2}$, which belonged to known quantities. The two former values in the matrix are temperature measurement results of temperature at certain time. Two rear values were change rates of temperature on time, which can be obtained by temperature measurement results and time step differential form. Therefore, polynomial equation coefficient $C n(t)$ on the left of expression equation (5) can be uniquely determined through known quantity and measurement results. Temperature distribution in one dimensional control volume and surface heat flow density under hypothesis of equation (1) can be obtained.

$$
q_{i n}=\left.\frac{\partial T}{\partial x}\right|_{x=0}=C_{1}(t)+C_{2}(t)
$$

Inverse problem calculation based on temperature polynomial distribution in literature research was apparently applicable to one-dimensional problem of various boundary conditions, including: one-end insulation, semi-infinite, limitation and other problems.

\section{Design of Heat Flux Sensor}

Sensitive elements of heat flux sensor included circumferential thermal insulation round metal rods. It was defined that one end surface of the slab can accept heat load and energy input, and the other end was responsible for energy output. Since equation (1) had high applicability to various boundary conditions, one-dimensional slab energy output end surface can be adiabatic, semi-infinitely broad and infinitely broad, it corresponded to heat insulating layer, heat sink structure, air/liquid cooling, etc.

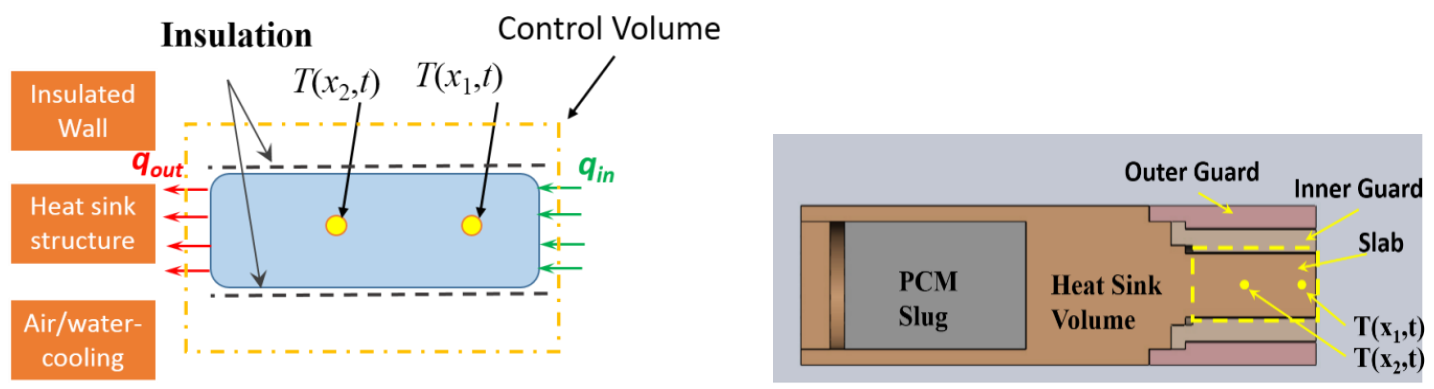

Fig.1 Domains addressed by Edward's theory[10] Fig.2 Schematic for heat flux sensor contains PCM 
Heat flux sensor structure included a variable cross-section oxygen-free copper slab with total length of $71 \mathrm{~mm}$, wherein the part with diameter of $\Phi 8 \times 18(\mathrm{~mm})$ was one-dimensional control volume of calculation model. After it cooperated with inner guard and outer guard through two orders, it formed heat sink volume of $\Phi 20 \times 48$, the area was emptied, one $\Phi 16 \times 25$ PCM slug was placed. The back end surface was sealed by bottom cover. Since the volume of alloy was expanded after being melted, gap of $\Phi 16 \times 2 \mathrm{~mm}$ was left between bottom cover and PCM slug. Gap of $0.15 \mathrm{~mm}$ was left between inner guard and slab as well as between outer guard and inner guard. Radial conduction on slabs can be minimized, thereby meeting one dimensional hypothesis of control volume. Two K-shaped thermocouples were placed on different positions along the axial direction for obtaining temperature time history of the two points.

\section{Preparation of PCM Slug}

In the paper, alloy (Bi, $\mathrm{Sn}-52 \%)$ was adopted as phase change material, $\mathrm{Sn}$ - Bi alloy phase change latent heat was $49.1 \mathrm{~kJ} / \mathrm{kg}$ with melting point of $138{ }^{\circ} \mathrm{C}$. Foam copper $(99.8 \%$ pure copper with density of $300 \mathrm{~kg} / \mathrm{m} 3$ and porosity of $97 \%$ ) was used as thermal conduction enhancement skeleton for producing PCM slug. Foam skeleton containing copper foam and alloy composite slug underwent simplified treatment in literature [11]. Performance characterization was implemented on $\mathrm{Sn}-52 \% \mathrm{Bi}$ alloy composite materials with foam copper as skeleton. Unit cell model was established according to geometrical characteristics of copper foam. Equivalent thermal conductivity of unit cell materials was obtained by numerical simulation. 'Equivalent specific heat capacity' of slug prepared by alloy under solid, phase change and liquid states was obtained. Finally, rationality of simplification method was verified through test. The above treatment methods and results were continuously cited in the paper.

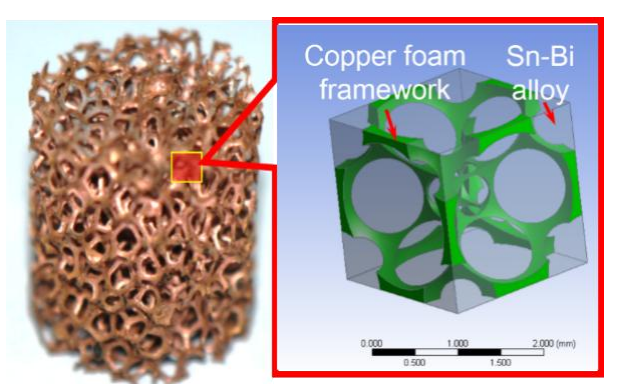

Fig.3 Copper foam cylinder and PCM slug unit slug cell model

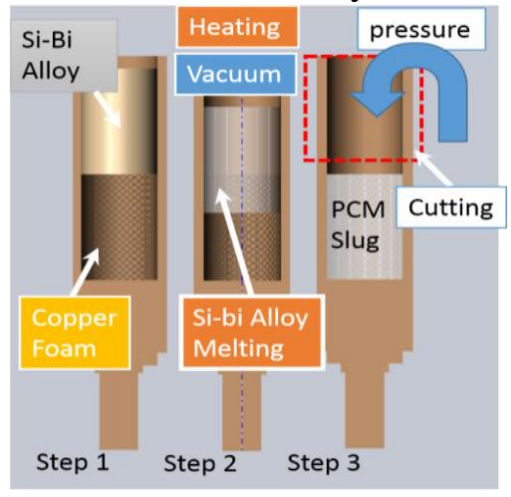

Fig.4 Schematic diagram of PCM slug fabrication

PCM slug was directly formed in sensor slab. Specific method was shown as follows. Holes were drilled in heat sink area of sensor round cross-section with diameter of $16 \mathrm{~mm}$ which was the same as the bubble copper and alloy rod materials. The slab was uprightly placed, foam copper was placed below alloy rod materials, which was placed into vacuum heating furnace as a whole. After the furnace was vacuumized, it was heated until alloy was melted completely. Inside of the furnace was punched to atmosphere pressure, and the alloy was completely immersed into grid gap of foam copper. Finally, excessive part of round rod length was cut.

\section{Test Design}

Halogen lamp equipment was adopted for constant heat flux sensor loading. Solid slab was adopted for preparing a sensor with consistent geometry size and without PCM for control comparison in order to verify energy storage function of metal phase change materials. Surfaces of two sensors were coated into black. Outer edges of sensor were wrapped by insulation materials in order to reduce interference of lateral heat transfer. Aluminum structure was wrapped outside the heat insulation materials, which was used for polishing the surface of heat source, thereby avoiding rapid temperature 
rise on the surface thereof under the load effect of heat insulation materials. The same boundary conditions were formed for two sensors in the test as far as possible.

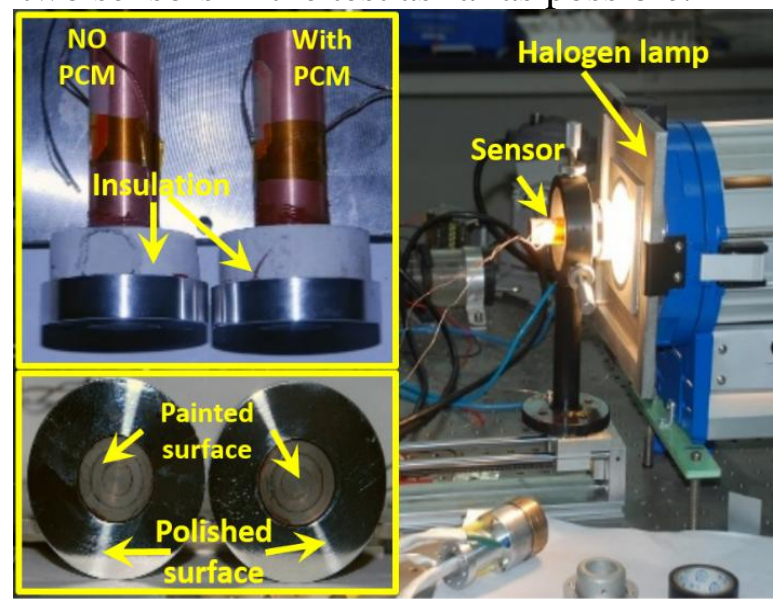

Fig.5 Sensors tested by halogen lamp facility

Heat flux projected to the specimen surface was adjusted in the distance between specimen and equipment according to power of halogen lamp equipment. Gardon Gage was used for calibrating heat flux, and calibration results were shown in the following table:

Tab. 1 Calibration results for halogen lamp equipment heat flux

\begin{tabular}{ccc}
\hline Facility power & $\begin{array}{c}\text { Distance between sensor and } \\
\text { facility }\end{array}$ & $\begin{array}{c}\text { heat flux(measured by } \\
\text { Gardon gage) }\end{array}$ \\
\hline $756[\mathrm{~W}]$ & $11.34[\mathrm{~mm}]$ & $206.50\left[\mathrm{KW} / \mathrm{m}^{2}\right]$ \\
\hline
\end{tabular}

Basic performances of prepared heat flux sensor containing PCM and common heat flux sensor as control were shown as follows:

Tab. 2 Performance of sensors for test

\begin{tabular}{ccc}
\hline Sensor properties & With PCM & NO PCM \\
\hline Slab weight $[\mathrm{m}]$ & $179.5[\mathrm{~g}]$ & $196.3[\mathrm{~g}]$ \\
TC\#1 location $\left[x_{1}\right]$ & $3.85[\mathrm{~mm}]$ & $3.75[\mathrm{~mm}]$ \\
TC\#2 location $\left[x_{2}\right]$ & $11.85[\mathrm{~mm}]$ & $11.79[\mathrm{~mm}]$ \\
& \multicolumn{2}{c}{ (TC: Thermocouple) } \\
\hline
\end{tabular}

\section{Results and Discussion}

Sensors containing PCM slug and control sensor were respectively loaded for 200S by halogen lamp. Temperature processes of two thermo-couples in all sensors were shown in the following figure. Two temperatures of the sensors were continuously increased in heating time, which were rapidly decreased after temperature rise stopped. Two temperature peaks of the sensor containing PCM respectively reached $157.6^{\circ} \mathrm{C}$ and $154.6^{\circ} \mathrm{C}$. Two temperature peaks of control sensor excluding PCM reached $157.1^{\circ} \mathrm{C}$ and $153.4^{\circ} \mathrm{C}$

Temperature processes of two sensors were respectively added into equation (5) - (7) for solving surface heat flux. The results were shown in the following figure. Deviation of measurement results of two sensors from Gordon Gage calibration value was $\pm 5 \%$. Sensitive principle and measurement functions of sensors were verified.

Heat flux measurement result corresponded to time integral and was multiplied by sensor sensitive area for obtaining total energy in the sensor (total heat quantity). Total heat quantity of sensor containing PCM core was $2157.93 \mathrm{~J}$, and the control sensor was $2158.69 \mathrm{~J}$. Function $K_{J}$ was constructed for characterization in order to further assess heat sink efficiency of sensor: ratio between heat absorbed by sensor unit mass and corresponding sensor temperature rise at the same time interval 
had dimension: $\mathrm{J} / \mathrm{C}^{\mathrm{o}} \cdot \mathrm{g} \cdot \mathrm{m}^{2}$, temperature rise was uniformly calculated with temperature of sensitive round rod surface $x=0$ of two sensors, and the surface temperature was obtained in the process of calculating heat flux in equation (5) (7). Surface temperature and heat sink efficiency ratio of sensor containing / excluding heat sink were shown in the following figure:

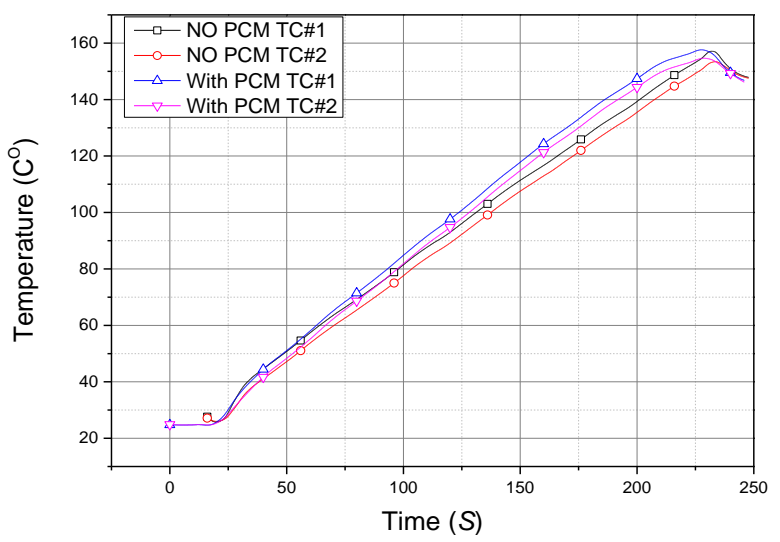

Fig.6 Temperatures measured by sensor's Thermocouple

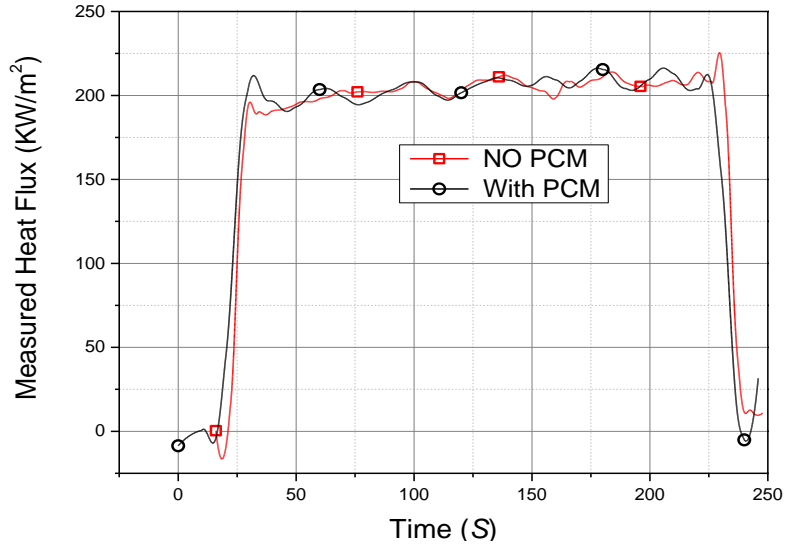

Fig.7 Heat flux calculated by measured temperature

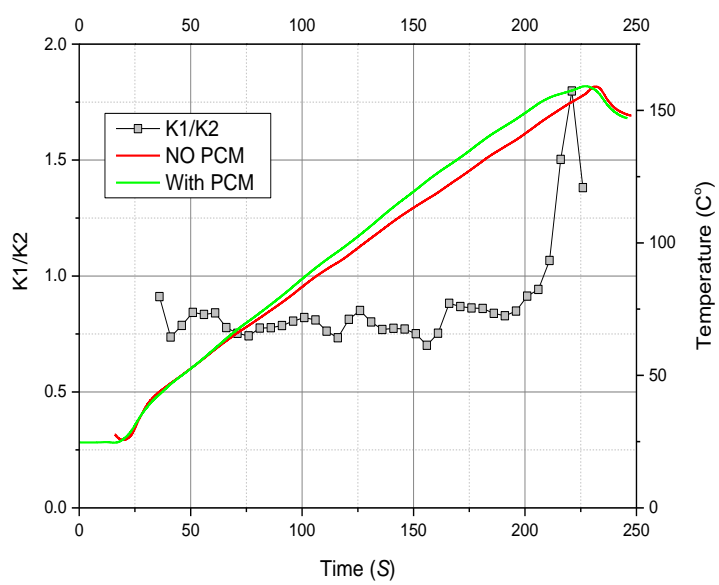

Fig.8 Heat flux sensor surface temperature calculated value and ratio to $K_{1}$ and $K_{2}$

Figure 8 showed that total temperature rise of sensor containing PCM and sensor excluding PCM was basically the same within the test time of 200S. After the test was started, the temperature rise speed of sensor containing PCM slug was faster than that of control sensor. Temperature on the surface of sensor containing PCM was increased to $150^{\circ} \mathrm{C}$ or so, the temperature rise speed was prominently slowed down, $\mathrm{Sn}-\mathrm{Bi}$ alloy inside corresponding sensor sensor slab began to melt (alloy melting point: $138{ }^{\circ} \mathrm{C}$ ). Energy in the sensor was converted into alloy phase change latent heat. Similarly, ratio between K1 and K2 showed that 'heat sink efficiency' of heat flux sensor containing PCM before alloy melting was lower than oxygen-free copper solid of control sensor, the ratio was about $0.8 \sim 0.8$, and the ratio rapidly exceeded 1 and increased to 1.7 or so.

\section{Summary}

The following conclusion were obtained in the study through stimulation on finite element and comparison, analysis and discussion with experimental results:

(1) PCM slug heat sink has prominently higher 'heat sink efficiency' compared with oxygen free copper in the alloy phase change stage. Heat flux measurement function was kept normal.

(2) Heat flux sensor containing PCM and control sensor have almost equal temperature rise under nearly the same energy input, but the quality of heat flux sensor containing PCM is lighter than the later by $16.8 \mathrm{~g}$. 
(3) Alloy with low melting point can be optimally designed in further research according to load type and measurement demand, thereby obtaining ideal phase change latent heat and melting point, and performance and shape of heat conduction enhancement skeleton can be optimally designed.

\section{Reference}

[1]W.K.P van Loona, H.M.H Bastingsa, E.J Moorsb: Agricultural and Forest Meteorology Vol. 92(1998), P: 1-8

[2]Bales, E., Bomberg, M., Courville, G. E: ASTM Report: ASTM 885, (1985).

[3]Imelda, Terrazas-Salinas, J. Enrique Carballo, et al: AIAA Report: AIAA 2010-5053, (2010).

[4]P R N Childs, J R Greenwood, C A Long: Proceedings of The Institution of Mechanical Engineers Part C-Journal Of Mechanical Engineering Science - PROC INST MECH ENG C-J MECH E Vol.213(1999), P: 655-677

[5]A. H. Epstein1, G. R. Guenette1, R. J. G. Norton, et al: Review of Scientific Instruments, 57(1986), P: 639

[6]Michael J. W, Frank S. M: Journal Of Spacecraft And Rockets Vol. 43(2006), P: 929-942.

[7]R Müller Eigner, G. Koppenwallner: Fourth Symposium on Aerothermodynamics for Space Vehicles. Capua: European Space Agency, (2002), P: 457-464.

[8]Turner J, Hörschgen M, Stamminger A, Turner P: AIAA Report: AIAA-2006-8115, (2006).

[9]Sharma A, Tyagi V V, Chen C R, et al: Renewable \& Sustainable Energy Reviews Vol. 13(2009), P: 318-345.

[10]Edward B. Coy: Journal Of Thermophysics And Heat Transfer Vol. 24, (2010), P: 77-84.

[11]Xiaoheng Ding, Songhe Meng, Weihua Xie, et al: Acta Materiae Compositae Sinica. Vol.30(2013), P: 205-211. 